\title{
Investigation of Surface Plasmon Coupling and Damping in Au and Ag Nanoparticle Assemblies by Monochromated Electron Energy Loss Spectroscopy.
}

\author{
A.M. Thron, A. Polyakov, P.J. Shuck, S. Aloni \\ Molecular Foundry, Lawrence Berkeley National Laboratory, One Cyclotron Road, Berkeley, CA \\ 94720
}

Surface plasmons confine light to nanostructures smaller than the diffraction limit by coupling the electromagnetic radiation with the oscillation of free charge carriers in the structure. The shape and local dielectric environment can alter the resonant behavior of the SPs, as well as intrinsic material properties such as chemistry and defects. Monochromated Scanning Transmission Electron Microscopy (STEM) and Electron Energy Loss Spectroscopy (EELS) has proven to be an effective technique in characterizing SP at the nanoscale [1]. Resistive and capacitive SP coupling is shown to occur between symmetric Au nanowire dimers [2]. Theoretical calculations have shown that breaking the symmetry parallel to the nanowire axes causes near field interference between the dimers [3]. However, few studies have experimentally investigated the near field interaction of adjacent nanoparticles which break the symmetry perpendicular to the rod axes. The aim of our study is characterize coupling and dampening of SPs in Au and Ag and Au nanorods, caused by neighboring particles which breaks the symmetry of the local dielectric environment.

$\mathrm{Au}$ and Ag nanoantennas rods are used as models systems since their shape dependent SP resonances are in the near infrared and the visible range of the spectrum, giving enough separation from the zero loss peak (ZLP) to be resolved spectroscopically. To study the spatial distribution of the of the plasmon resonances we acquired a hyperspectral EELS map of nanoparticle assemblies. To further improve the accuracy of the analysis the ZLP tail has been subtracted by fitting an analytical peak function (Voigt) [4], and the spatial distribution of the individual resonant modes are compared to that of references taken form individual nanoparticles. The nanocube appears to break the symmetry of each SP mode accept for the strictly confined surface mode at $3.3 \mathrm{eV}$ (Figure 1). For example, the second SP mode in Figure $2 \mathrm{c}$ has three nodes which are degenerate. Comparing the same surface plasmon mode for the rod-cube pair, the bottom node shifts by $0.19 \mathrm{eV}$. This break in degeneracy is caused by the near field coupling of the Ag nanorod and Ag nanocube.

This breaking symmetry and reduced degeneracy is also manifested in the broadening of the SP peaks occurring in the assemblies. The full width half maximum (FWHM) of the SP peaks were measured by fitting a Gaussian to the measured peaks. Peaks were measured along the side of the rod, rather than directly over the rod, to rule out extra broadening caused by the sample. Except for the first mode, the width of the SP peak is increased by at least $0.2 \mathrm{eV}$ when compare with single rods (Figure 2). An increase in SP peak width is attributed to change in the damping of individual SP resonances, and is observed in other nanoparticle assemblies. However, understanding the nature of this phenomenon is not straight forward. The spatial distribution of SP mode intensities will require detailed analytical and theoretical modeling. We will discuss these results in context of FDTD simulations that are expected to describe in detail the coupling between the SP modes of the assembly components.

\section{References}

[1] Rossouw D., Couillard M, Vickery J., Kumacheva E. Botton G.A., Nano Lett., 11 (2011), 1499-1504 
[2] Alber I. et. al., ACS Nano, 6 (2012), 9711-9717

[3] Bigelow N.W., Vaschillo A., Camden J.P., Masiello D.J., ACS Nano, 7 (2013), 4511-4519

[4] Hyperspectral Data Analysis Software, http://hyperspy.org/
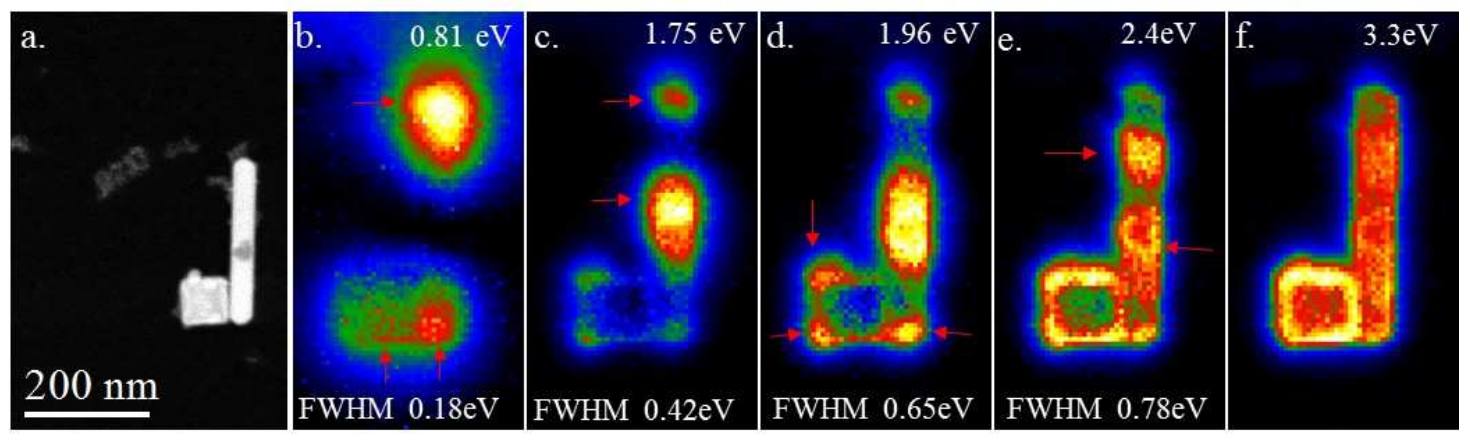

Figure 1. (a) Annular Dark Field image of adjacent Ag nanoantenna and nanocube. (b-e) Spectrum maps of the separate SP peaks which appear in the energy loss spectrum. (f) SP which is strictly confined to the surface of the nanoantenna and nanocube. Each map is labeled by its peak position and the FWHM measured from a Gaussian fit. Due to the overlap in SP peaks, spectral intensities give the false appearance of SP nodes. Therefore, red arrows highlight the location of the degenerate nodes.
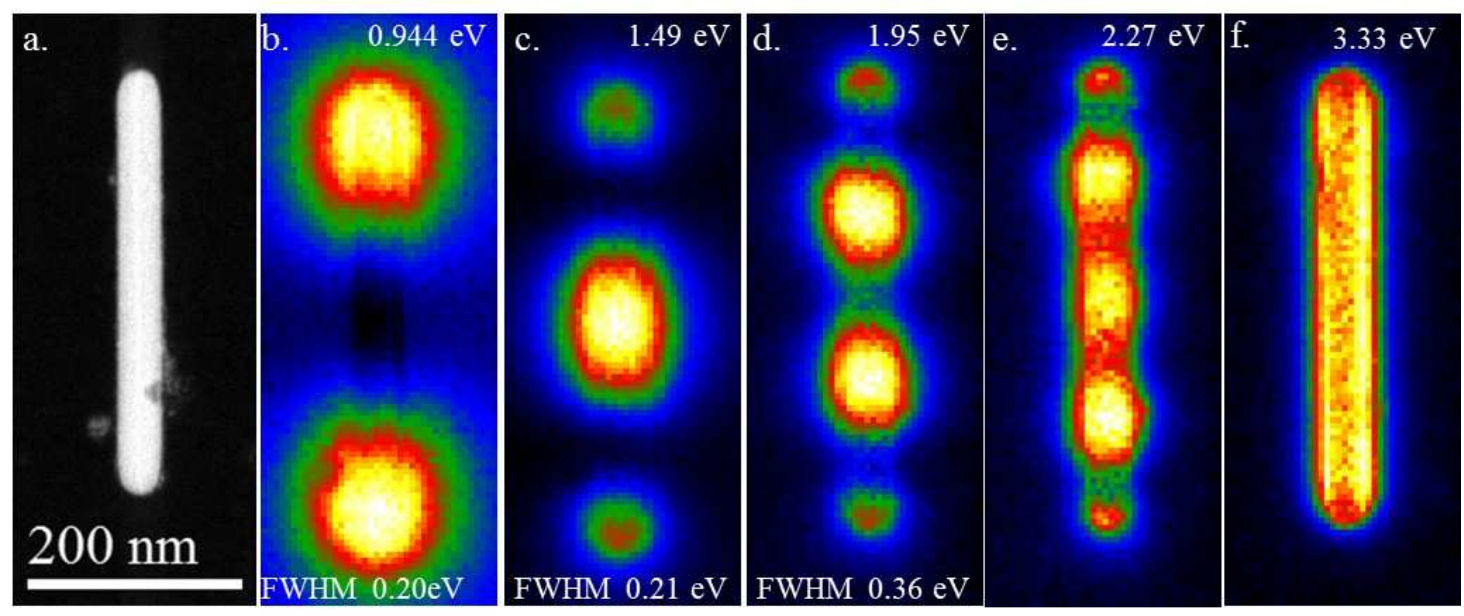

Figure 2. (a) Annular Dark Field image of a lone Ag nanoantenna (b-e) Spectrum maps of the separate SP peaks which appear in the energy loss spectrum. (f) SP which is strictly confined to the surface of the nanoantenna. Each map is labeled by its peak position and the FWHM measured from a Gaussian fit. The FWHM is not measured in (e) due the strong overlap with other peaks. 